PROCEEDINGS OF THE

AMERICAN MATHEMATICAL SOCIETY

Volume 129, Number 3, Pages 873-879

S 0002-9939(00)05821-4

Article electronically published on October 11, 2000

\title{
ORTHOGONAL POLYNOMIALS ON THE UNIT CIRCLE ASSOCIATED WITH THE LAGUERRE POLYNOMIALS
}

\author{
LI-CHIEN SHEN
}

(Communicated by Hal L. Smith)

\begin{abstract}
Using the well-known fact that the Fourier transform is unitary, we obtain a class of orthogonal polynomials on the unit circle from the Fourier transform of the Laguerre polynomials (with suitable weights attached). Some related extremal problems which arise naturally in this setting are investigated.
\end{abstract}

\section{INTRODUCTION}

This paper deals with a class of orthogonal polynomials which arise from an application of the Fourier transform on the Laguerre polynomials.

We shall briefly describe the essence of our method.

Let $\Pi^{+}$denote the upper half plane $\{z: z=x+i y, y>0\}$ and let

$$
H\left(\Pi^{+}\right)=\left\{f: f \text { is analytic in } \Pi^{+} \text {and } \sup _{0<y<\infty} \int_{-\infty}^{\infty}|f(x+y i)|^{2} d x<\infty\right\} .
$$

It is well known that, from the Paley-Wiener Theorem 4, p. 368], the Fourier transform provides a unitary isometry between the spaces $L^{2}(0, \infty)$ and $H\left(\Pi^{+}\right)$. Since the Laguerre polynomials form a complete orthogonal basis for

$$
L^{2}\left([0, \infty), x^{\alpha} e^{-x} d x\right),
$$

the application of Fourier transform to the Laguerre polynomials (with suitable weight attached) generates a class of orthogonal rational functions which are complete in $H\left(\Pi^{+}\right)$; and by composition of which with the fractional linear transformation (which maps $\Pi^{+}$conformally to the unit disc)

$$
z=(2 t-i) /(2 t+i),
$$

we obtain a family of polynomials which are orthogonal with respect to the weight $\sin ^{\alpha} \frac{t}{2} d t$ on the boundary $|z|=1$ of the unit disc.

Some similar approaches of linking the classical orthogonal polynomials with orthoganal polynomials of greater generality via various transforms have been investigated recently. For example, using the Fourier transform, Koelink [1] established the connection between the Jacobi polynomials and the continuous Hahn polynomials; and in 2] Koornwinder established the link between the Meixner-Polleczek polynomials and the Laguerre polynomials from the Mellin transform.

Received by the editors June 1, 1999.

2000 Mathematics Subject Classification. Primary 33C47.

Key words and phrases. Fourier transform, Laguerre polynomial.

(C)2000 American Mathematical Society 
We thank the referee for many detailed suggestions in revising the original manuscript as well as for drawing our attention to papers [1] and [2].

\section{KEY RESUlts}

We assume that the reader is reasonably familiar with the basic properties of the Laguerre polynomials which can be found in Chapter 4 of 3 .

We say a few words about our notation. The Fourier transform of an integrable function $f$ is defined as

$$
\hat{f}(t)=\int_{-\infty}^{\infty} e^{i x t} f(x) d x
$$

$(a)_{n}=a(a+1)(a+2) \ldots(a+n-1)$ and $F(a, b ; c ; z)$ is the standard hypergeometric series.

Let

$$
L_{n}^{\alpha}(x)=\frac{(\alpha+1)_{n}}{n !} \sum_{k=0}^{n} \frac{(-n)_{k} x^{k}}{k !(\alpha+1)_{k}} \quad(\alpha>-1)
$$

be the Laguerre polynomial of degree $n$ associated with the weight $e^{-x} x^{\alpha} d x$, and let

$$
l_{n}^{\alpha}(x)= \begin{cases}L_{n}^{\alpha}(x) e^{-x / 2} x^{\alpha / 2}, & x \geq 0, \\ 0, & x<0 .\end{cases}
$$

We now compute the Fourier transform of $l_{n}^{\alpha}(x)$. First, we observe that

$$
\begin{gathered}
\hat{l}_{0}^{\alpha}(t)=\int_{0}^{\infty} e^{i x t} e^{-x / 2} x^{\alpha / 2} d x=\Gamma\left(\frac{\alpha}{2}+1\right)\left(\frac{1}{2}-i t\right)^{-\frac{\alpha}{2}-1} \\
(-i D)^{n} \hat{l}_{0}^{\alpha}(t)=\int_{0}^{\infty} e^{i x t} e^{-x / 2} x^{\alpha / 2+n} d x=\left(\frac{\alpha}{2}+1\right)_{n}\left(\frac{1}{2}-i t\right)^{-n} \hat{l}_{0}^{\alpha}(t),
\end{gathered}
$$

where $(-i D)^{n}=(-i)^{n} \frac{d^{n}}{d t^{n}}$ and $t$ is a complex number with imaginary part $>-\frac{1}{2}$. It is easy to justify the differentiation behind the integral sign because of the absolute convergence of the integral. From (2.1) and (2.2), we obtain

$$
\begin{aligned}
\hat{l}_{n}^{\alpha}(t) & =\int_{0}^{\infty} e^{i x t} x^{\alpha / 2} e^{-x / 2} L_{n}^{\alpha}(x) d x=L_{n}^{\alpha}(-i D) \hat{l}_{0}^{\alpha}(t) \\
& =\frac{(1+\alpha)_{n}}{n !} \hat{l}_{0}^{\alpha}(t) \sum_{k=0}^{n} \frac{(-n)_{k}\left(\frac{\alpha}{2}+1\right)_{k}}{k !(\alpha+1)_{k}}\left(\frac{1}{\frac{1}{2}-i t}\right)^{k} \\
& =\frac{(1+\alpha)_{n}}{n !} \hat{l}_{0}^{\alpha}(t) F\left(-n, \frac{\alpha}{2}+1 ; \alpha+1 ; \frac{1}{\frac{1}{2}-i t}\right) .
\end{aligned}
$$

Now let $z=(2 t-i) /(2 t+i)$ and recall that

$$
F(-n, a ; c ; x)=\frac{(c-a)_{n}}{(c)_{n}} F(-n, a ; a-n-c+1 ; 1-x) .
$$

We can write

$$
\begin{aligned}
\hat{l}_{n}^{\alpha}(t) & =\Gamma\left(\frac{\alpha}{2}+1\right) \frac{(1+\alpha)_{n}}{n !}(1-z)^{\frac{\alpha}{2}+1} F\left(-n, \frac{\alpha}{2}+1 ; \alpha+1 ; 1-z\right) \\
& =\Gamma\left(\frac{\alpha}{2}+1\right)(1-z)^{\frac{\alpha}{2}+1} g_{n}^{\alpha}(z),
\end{aligned}
$$


where

$$
\begin{aligned}
g_{n}^{\alpha}(z) & =\frac{(\alpha / 2)_{n}}{n !} F\left(-n, \frac{\alpha}{2}+1 ;-n-\frac{\alpha}{2}+1 ; z\right) \\
& =\frac{(1+\alpha)_{n}}{n !} F\left(-n, \frac{\alpha}{2}+1 ; \alpha+1 ; 1-z\right)
\end{aligned}
$$

is a polynomial of degree $n$. For brevity, we let $g_{n}(z)=g_{n}^{\alpha}(z)$. We choose the branch of $(1-z)^{\frac{\alpha}{2}+1}$ which takes the value 1 at $z=0$. Therefore $\hat{l}_{n}^{\alpha}$, as a function of $z$, is analytic in the complex plane cut along $[1, \infty)$. Moreover, from the fact that the Fourier transform preserves the inner product (the Parseval's identity), we deduce that

$$
\begin{aligned}
2^{\alpha} & \frac{\Gamma^{2}\left(\frac{\alpha}{2}+1\right)}{2 \pi} \int_{0}^{2 \pi} g_{n}\left(e^{i \theta}\right) \overline{g_{m}\left(e^{i \theta}\right)} \sin ^{\alpha} \frac{\theta}{2} d \theta \\
& =\frac{\Gamma^{2}\left(\frac{\alpha}{2}+1\right)}{2 \pi i} \int_{|z|=1} g_{n}(z) \overline{g_{m}(z)}|1-z|^{\alpha} \frac{d z}{z} \\
& =-\frac{\Gamma^{2}\left(\frac{\alpha}{2}+1\right)}{2 \pi i} \int_{|z|=1} g_{n}(z) \overline{g_{m}(z)}(1-z)^{\frac{\alpha}{2}+1}(1-\bar{z})^{\frac{\alpha}{2}+1}(1-z)^{-2} d z \\
& =\frac{1}{2 \pi} \int_{-\infty}^{\infty} \hat{l}_{n}^{\alpha}(t) \overline{\hat{l}_{m}^{\alpha}(t)} d t \\
& =\int_{0}^{\infty} l_{n}^{\alpha}(x) \overline{l_{m}^{\alpha}(x)} d x \quad(\text { Parseval's identity) } \\
& =\int_{0}^{\infty} L_{n}^{\alpha}(x) L_{m}^{\alpha}(x) e^{-x} x^{\alpha} d x \\
& =\left\{\begin{array}{c}
\frac{\Gamma(n+\alpha+1)}{n !} \quad n=m \\
0 \quad n \neq m .
\end{array} \quad([3, \text { p. 84]). }\right.
\end{aligned}
$$

We summarize our result in

Theorem 1. The polynomials $\left\{g_{n}^{\alpha}(z)\right\}_{n=0}^{\infty}$ form a complete orthogonal basis for

$$
H_{\mu}^{2}=\left\{f: f \text { is analytic in }|z|<1 \text { and } \int_{0}^{2 \pi}\left|f\left(e^{i \theta}\right)\right|^{2} d \mu<\infty\right\} \text {, }
$$

where $d \mu=\frac{1}{2 \pi} \sin ^{\alpha} \frac{\theta}{2} d \theta$.

The completeness follows immediately from the completeness of $l_{n}^{\alpha}(x)$ in $L^{2}[0, \infty)$ [5, p. 108], the Paley-Wiener Theorem and the fact that the Fourier transform preserves the basis.

It is interesting to note that for $\alpha=0, g_{n}(z)=z^{n}$. And for $\alpha=2$,

$$
g_{n}(z)=\sum_{k=0}^{n}(k+1) z^{n}
$$

which is the $n$th partial sum of the familiar power series

$$
(1-z)^{-2}=\sum_{k=0}^{\infty}(k+1) z^{k}
$$


Remark 1 . We note that for $\alpha \geqslant 0,(-n)_{k} /\left(-n-\frac{\alpha}{2}+1\right)_{k} \geqslant 0$ for $n \geqslant k \geqslant 0$. Hence, from (2.3), we conclude that

$$
\max _{|z| \leqslant R}\left|g_{n}(z)\right|=g_{n}(R) .
$$

Similarly,

$$
\max _{|z| \leqslant R}\left|g_{n}^{(k)}(z)\right|=g_{n}^{(k)}(R)
$$

In particular,

$$
g_{n}(1)=(\alpha+1)_{n} / n ! \quad \text { and } \quad g_{n}^{(k)}(1)=\left(\frac{\alpha}{2}+1\right)_{k}(\alpha+1)_{n} /\left((\alpha+1)_{k}(n-k) !\right) .
$$

Remark 2. The reproducing kernel $K_{N}(z, w)$ with respect to the measure $d \mu$ for the polynomials of degree $\leqslant N$ is defined as

$$
K_{N}(z, w)=\sum_{n=0}^{N} c_{n} g_{n}(z) \overline{g_{n}(w)},
$$

where

$$
c_{n}=a_{\alpha} n ! /(\alpha+1)_{n} \quad \text { and } \quad a_{\alpha}=2^{\alpha} \Gamma^{2}\left(\frac{\alpha}{2}+1\right) / \Gamma(\alpha+1) .
$$

The significance of the term "reproducing kernel" will be made clear in the next section. Then, from the above remark, we have

$$
\max _{|w| \leqslant 1} K_{N}(w, w)=K_{N}(1,1)=a_{\alpha} \sum_{n=0}^{N}(\alpha+1)_{n} / n !
$$

More generally, let

$$
M_{N, k}(w)=\sum_{n=k}^{N} c_{n}\left|g_{n}^{(k)}(w)\right|^{2} .
$$

Then

$$
\max _{|w| \leqslant R} M_{N, k}(w)=M_{N, k}(R) .
$$

\section{Extremal PRoblems}

Let $P_{N}$ be the set of all polynomials of degree $\leqslant N$. It follows from the orthogonality (2.4) that if $p \in P_{N}$, then

$$
p(w)=\int_{|z|=1} \overline{K_{N}(z, w)} p(z) d \mu .
$$

More generally, by differentiating the above identity $k$ times, we have

$$
p^{(k)}(w)=\int_{|z|=1} \overline{\bar{D}^{k} K_{N}(z, w) p}(z) d \mu \quad\left(\bar{D}=\frac{\partial}{\partial \bar{w}}\right) .
$$

Applying the Schwarz inequality to (3.1), we deduce (with the help of orthogonality)

$$
\left|p^{(k)}(w)\right| \leqslant\|p\|_{\mu} M_{N, k}(w) \quad \text { for } p \in P_{N},
$$

where $\|p\|_{\mu}^{2}=\int_{|z|=1}|p(z)|^{2} d \mu$. 
Therefore, we have

Theorem 2. If $p \in P_{N}$, then

$$
\sup _{\|p\|_{\mu}=1}\left|p^{(k)}(w)\right|=M_{N, k}(w),
$$

and the equality is achieved by the polynomial

$$
p(z)=\sum_{n=k}^{N} c_{n} g_{n}(z) \overline{g_{n}^{(k)}(w)} / M_{N, k}(w) .
$$

We comment that this extremal function $p(z)$ is essentially unique. To see this, we recall that [2, p. 63] in Schwarz inequality

$$
|(f, g)| \leq\|f\|\|g\|,
$$

the equality holds if and only if $f=c g$ a.e. Hence, the equality in (3.2) holds if and only if $p(z)=c \bar{D}^{k} K_{N}(z, w)$; and $c=a / M_{N, k}(w),|a|=1$, if we further require that $\|p\|_{\mu}=1$.

Corollary. If $p \in P_{N}$ and $\|p\|_{\mu}=1$, then

$$
|p(w)| \leqq 2^{\alpha} \frac{\Gamma\left(\frac{\alpha}{2}+1\right)}{\Gamma(\alpha+1)} \cdot \sum_{n=0}^{N} \frac{(\alpha+1)_{n}}{n !}
$$

for $|w|=1$.

From (3.2), we can also establish that if $p \in P_{N}$, then

$$
\inf _{\|p(k)\|_{\infty}=1}\|p\|_{\mu}=1 / M_{N, k}(R),
$$

where $\left\|p^{(k)}\right\|_{\infty}=\max _{|z|=R}\left|p^{(k)}(z)\right|$.

The proof is simple and it goes as follows. Choose a point $w$ on the circle $|z|=R$, so that $\left|p^{(k)}(w)\right|=\left\|p^{(k)}\right\|_{\infty}=1$. Then, according to (3.2) and Remark 2,

$$
\|p\|_{\mu} \geqslant 1 / M_{N, k}(w) \geqslant 1 / M_{N, k}(R),
$$

and again the equality holds for

$$
p(z)=\sum_{n=k}^{N} c_{n} g_{n}(z) \overline{g_{n}^{(k)}(R)} / M_{N, k}(R) .
$$

We remark that the extremal problems (the Bernstein-Markoff type inequalities) concerning the norm of the derivatives of a polynomial subject to certain given constraint have been studied by numerous authors and some of the more recent results of this nature have appeared in [6] and 7].

\section{Connection with the Gegenbauer polynomials}

To see the connection of $g_{n}^{\alpha}(z)$ with the Gegenbauer polynomial $C_{n}^{\lambda}(x)$, we write $\left(-n-\frac{\alpha}{2}+1\right)_{k}=(-1)^{k}(\alpha / 2)_{n} /(\alpha / 2)_{n-k}$; then

$$
g_{n}^{\alpha}(z)=\sum_{k=0}^{n} \frac{\left(\frac{\alpha}{2}+1\right)_{k}\left(\frac{\alpha}{2}\right)_{n-k}}{k !(n-k) !} z^{k} .
$$


We recall that the definition for the generating function for $C_{n}^{\lambda}(\cos \theta)$ is

$$
\sum_{n=0}^{\infty} C_{n}^{\lambda}(\cos \theta) r^{n}=1 /\left(1-2 r \cos \theta+r^{2}\right)^{\lambda} .
$$

Now write

$$
\left(1-r e^{i \theta}\right)^{-1}\left(1-2 r \cos \theta+r^{2}\right)^{-\frac{\alpha}{2}}=1 /\left(1-r e^{-i \theta}\right)^{\frac{\alpha}{2}}\left(1-r e^{i \theta}\right)^{\frac{\alpha}{2}+1} .
$$

Then the series expansion for the left-hand side is

$$
\left(\sum_{n=0}^{\infty} e^{i n \theta} r^{n}\right)\left(\sum_{n=0}^{\infty} C_{n}^{\alpha / 2}(\cos \theta) r^{n}\right)=\sum_{n=0}^{\infty} r^{n} e^{i n \theta} \sum_{k=0}^{n} C_{k}^{\alpha / 2}(\cos \theta) e^{-i k \theta},
$$

and the series expansion for the right-hand side is

$$
\begin{aligned}
& \left(\sum_{n=0}^{\infty} \frac{\left(\frac{\alpha}{2}+1\right)_{n}}{n !} r^{n} e^{i n \theta}\right)\left(\sum_{n=0}^{\infty} \frac{\left(\frac{\alpha}{2}\right)_{n}}{n !} r^{n} e^{-i n \theta}\right) \\
& =\sum_{n=0}^{\infty} r^{n} e^{-i n \theta} \sum_{k=0}^{n} \frac{\left(\frac{\alpha}{2}+1\right)_{k}\left(\frac{\alpha}{2}\right)_{n-k}}{k !(n-k) !} e^{2 i k \theta}=\sum_{n=0}^{\infty} r^{n} e^{-i n \theta} g_{n}^{\alpha}\left(e^{2 i \theta}\right) .
\end{aligned}
$$

We note that the series are absolutely convergent for $|r|<1$.

Equating the corresponding terms in both series, we have

$$
g_{n}^{\alpha}\left(e^{2 i \theta}\right)=\sum_{k=0}^{n} C_{k}^{\alpha / 2}(\cos \theta) e^{(2 n-k) i \theta} .
$$

We can derive a second relation with the Gegenbauer polynomials by writing

$$
\begin{aligned}
(1 & \left.-r e^{i \theta}\right)^{-1}\left(1-2 r \cos \theta+r^{2}\right)^{-\alpha / 2}=\left(1-r e^{-i \theta}\right)\left(1-2 r \cos \theta+r^{2}\right)^{-\alpha / 2-1} \\
& =\left(1-r e^{-i \theta}\right) \sum_{n=0}^{\infty} C_{n}^{\frac{\alpha}{2}+1}(\cos \theta) r^{n} \\
& =C_{0}^{\frac{\alpha}{2}+1}(\cos \theta)+\sum_{n=1}^{\infty}\left(C_{n}^{\frac{\alpha}{2}+1}(\cos \theta)-e^{-i \theta} C_{n-1}^{\frac{\alpha}{2}+1}(\cos \theta)\right) r^{n} .
\end{aligned}
$$

Then

$$
g_{n}^{\alpha}\left(e^{2 i \theta}\right)=e^{i n \theta}\left(C_{n}^{\frac{\alpha}{2}+1}(\cos \theta)-e^{i \theta} C_{n-1}^{\frac{\alpha}{2}+1}(\cos \theta)\right),
$$

and

$$
g_{0}^{\alpha}\left(e^{2 i \theta}\right)=C_{0}^{\frac{\alpha}{2}+1}(\cos \theta)
$$

\section{REFERENCES}

1. H. T. Koelink, On Jacobi and continuous Hahn polynomials, Proc. Amer. Math. Soc. 124 (1996), no.3, 887-898. MR 96f:33018

2. T. H. Koornwinder, Meixner-Pollaczek polynomials and Heisenberg algebra, J. Math. Phys. 30 (1989), no.4, 767-769. MR 90e:33037

3. N. N. Lebedev, Special Functions and Their Applications, Dover Publications, Inc., New York (1972). MR 50:2568

4. W. Rudin, Real and Complex Analysis, McGraw-Hill, New York (1966). MR 35:1420

5. G. Szego, Orthogonal Polynomials, American Mathematical Society, New York (1959). MR 21:5029 
6. A. K. Varma, Markoff type inequalities for curved majorants is $L^{2}$ norm in approximation theory (Kecskemet, 1990),689-697, Colloq. Math. Soc. Janos Bolyai, 58, North-Holland, Amsterdam, 1991. MR 94e:26028

7. A. K. Varma, On some extremal properties of algebraic polynomials, J. Approx. Theory 69 (1992), 48-54. MR 92m:41042

Department of Mathematics, University of Florida, Gainesville, Florida 32611-8105

E-mail address: shen@math.ufl.edu 